\title{
Relato de Experiência: Projeto de Pesquisa Anatomia nas Escolas realizado no município de Vassouras
}

\author{
Experience Report: Anatomy in Schools Research Project held in Vassouras Municipality \\ A Informe de experiencia: Proyecto de investigación sobre anatomía en las escuelas realizado en el \\ municipio de Vassouras
}

\author{
Juliana Profilo Sampaio ${ }^{1}$, Fabiane Roberto Leite ${ }^{2}$, Karine Grillo de Freitas ${ }^{3}$, Julia da Silveira Pacheco \\ Ferraz $^{4}$, Valdir Donizeti Alves Junior ${ }^{5}$, Emílio Conceição de Siqueira ${ }^{6}$
}

Como citar esse artigo. Sampaio, JP; Leite, FR; de Freitas, KG; Ferraz, JSP; Junior, VDA; de Siqueira, EC. Relato de Experiência: Projeto de Pesquisa Anatomia nas Escolas realizado no município de Vassouras. Revista PróUniverSUS. 2019 Jul/Dez.; 10 (2): 2427.

\section{Resumo}

Há uma grande dificuldade no ensino escolar brasileiro, que é a correlação de conteúdos teóricos abordados, com a prática referente ao mesmo, principalmente quando se trata do ensino da anatomia humana. Isso ocorre devido à falta de recursos para a construção de laboratórios nas escolas, ou mesmo aquelas que já possuem, faltam recursos para sua manutenção e para o estímulo dos professores a desempenharem tal função nas escolas. O projeto Anatomia nas Escolas propõe interligar os conhecimentos teóricos e práticos no ambiente escolar, visando uma melhor compreensão sobre o corpo humano, a localização dos órgãos, suas principais funções e correlações clínicas relevantes a respeito de doenças e hábitos de vida prejudiciais à saúde. Neste estudo relatamos a experiência da Liga de Anatomia no primeiro trimestre de 2019 no projeto Anatomia nas Escolas, no qual realizamos atividades com encontros mensais no Colégio Estadual Centenário. A metodologia utilizada consiste, em um primeiro momento, na aplicação de um pré-teste, com perguntas relacionadas ao sistema que será abordado e, no segundo momento, no desenvolvimento de aulas teóricas e práticas. Após ser administrado a aula, é aplicado um pós-teste com questões equivalentes a primeira avaliação para analisar o desempenho que os alunos obtiveram. Ao final, fazemos uma dinâmica, com perguntas e respostas utilizando um manequim e órgãos plastificados. Desse modo, o objetivo do projeto é através do ensino prático consolidar o conhecimento dos sistemas do corpo humano ensinado nas escolas e promover o cuidado em saúde dos alunos no município de Vassouras.

Palavras-chave: Anatomia humana, Saúde, Ensino prático.

\begin{abstract}
There is a great difficulty in Brazilian school education, which is the correlation of theoretical contents approached with the practice related to it, especially when it comes to teaching human anatomy. This is due to the lack of resources to build laboratories in schools, or even those that already have them, lack resources to maintain them and to encourage teachers to perform such a function in schools. The Anatomy in Schools project proposes to link theoretical and practical knowledge in the school environment, aiming at a better understanding of the human body, the location of organs, their main functions and relevant clinical correlations regarding diseases and harmful lifestyle habits. In this study we report the experience of the Anatomy League in the first quarter of 2019 in the Anatomy in Schools project, in which we conducted activities with monthly meetings at the Centennial State College.The methodology used consists, initially, in the application of a pretest, with questions related to the system that will be addressed and, in the second moment, in the development of theoretical and practical classes. After the class is administered, a post-test with questions equivalent to the first assessment is applied to analyze the performance that students obtained. In the end, we make a dynamic, with questions and answers using a dummy and plasticized organs. Thus, the objective of the project is through practical teaching to consolidate the knowledge of human body systems taught in schools and promote health care for students in the municipality of Vassouras.
\end{abstract}

Keywords: Human anatomyhealth, Practical teaching.

2. Acadêmica do Curso de Medicina, Universidade de Vassouras. RJ, Brasil. Email: fabianeleite tp@hotmail.com ORCID: https://orcid.org/0000-0002-1019-0958

3. Acadêmica do Curso de Medicina, Universidade de Vassouras. RJ, Brasil. Email: karinegrillo@live.com ORCID: https://orcid.org/0000-0002-0011-4185

4. Acadêmica Acadêmico do Curso de Medicina, Universidade de Vassouras. RJ, Brasil. Email: juliaspf@gmail.com ORCID: https://orcid.org/0000-0001-5082-1294

5. Acadêmico Acadêmico do Curso de Medicina, Bolsista de Inciação Científica/CNPq. Universidade de Vassouras. RJ, Brasil. Email: valdir-alvesjr@hotmail.com ORCID: https:// orcid.org/0000-0001-7937-9671

6. Docente do Curso de Medicina, Universidade de Vassouras. RJ, Brasil. Email: emiliouba@uol.com.br ORCID: https://orcid.org/0000-0002-8489-6531 


\section{Resumen}

Hay una gran dificultad en la educación escolar brasileña, que es la correlación de los contenidos teóricos abordados con la práctica relacionada con ella, especialmente cuando se trata de enseñar anatomía humana. Esto se debe a la falta de recursos para construir laboratorios en las escuelas, o incluso aquellos que ya los tienen, carecen de recursos para mantenerlos y para alentar a los maestros a desempeñar esa función en las escuelas. El proyecto Anatomy in Schools propone vincular los conocimientos teóricos y prácticos en el entorno escolar, con el objetivo de comprender mejor el cuerpo humano, la ubicación de los órganos, sus funciones principales y las correlaciones clínicas relevantes con respecto a las enfermedades y los hábitos de vida perjudiciales. En este estudio, informamos la experiencia de Anatomy League en el primer trimestre de 2019 en el proyecto Anatomy in Schools, en el que realizamos actividades con reuniones mensuales en el CentennialStateCollege. La metodología utilizada consiste, inicialmente, en la aplicación de una prueba preliminar, con preguntas relacionadas con el sistema que se abordarán y, en el segundo momento, en el desarrollo de clases teóricas y prácticas. Después de que se administra la clase, se aplica un examen posterior con preguntas equivalentes a la primera evaluación para analizar el rendimiento que obtuvieron los estudiantes. Al final, hacemos una dinámica, con preguntas y respuestas utilizando un órgano ficticio y plastificado. Por lo tanto, el objetivo del proyecto es a través de la enseñanza práctica para consolidar el conocimiento de los sistemas del cuerpo humano que se enseñan en las escuelas y promover la atención médica para los estudiantes en el municipio de Vassouras.

Palabras clave: Anatomía humana, Salud, Docencia práctica.

\section{Introdução}

Educação em saúde pode ser definida como um conjunto de conhecimentos e práticas direcionadas para a prevenção de doenças e promoção da saúde. Para que isso ocorra de forma otimizada é imprescindível a junção entre Universidade e comunidade através de projetos de extensão. Trata-se de um recurso por meio do qual o conhecimento cientificamente produzido no campo da saúde, intermediado pelos acadêmicos da área saúde, atinge a vida cotidiana dos sujeitos, uma vez que a compreensão dos condicionantes do processo saúde-doença oferece subsídios para a adoção de novos hábitos e condutas de saúde ${ }^{1}$. A dimensão educativa objetiva contribuir na consolidação das informações prévias que os alunos do ensino fundamental possuem a respeito da anatomia do corpo humano e suas afecções, além de promover a educação em saúde valorizando a história de vida e permitir que os estudantes sejam sujeito do processo de aprendizado ${ }^{2}$.

O ensino de saúde é um grande desafio que busca garantir um conhecimento efetivo e transformador para o indivíduo ${ }^{3}$. É necessário, portanto, que nas escolas haja um incentivo ao ensino preventivo, promovendo o autocuidado da população através de divulgações de informações sobre hábitos de vida saudáveis. Desse modo, ensinar sobre o conhecimento da anatomia humana e o seu funcionamento representa um grande passo para a conscientização e a promoção da saúde na sociedade ${ }^{3}$. Além disso, é fundamental associar a Universidade a escola, afim de permitir uma melhor comunicação e divulgação do conhecimento aprendido na Universidade a uma parcela maior da população. Nota-se, ainda uma grande dificuldade de infraestrutura das escolas com relação ao ensino prático, uma vez que não há laboratórios, equipamentos e nem profissionais capacitados para atuar na prática do ensino ${ }^{4}$.

O projeto de pesquisa "Anatomia nas Escolas" desenvolvido pela Liga acadêmica de Anatomia Humana Professor Fróes da Fonseca da Universidade de Vassouras, aprovado no CEP, com parecer 2.919.792 propõe uma experiência diversificada no ensino do corpo humano. Esse projeto apresenta uma forma dinâmica e prática na abordagem dos principais sistemas do corpo, buscando através das peças do Instituto de Anatomia da Universidade de Vassouras conscientizar os alunos sobre as principais modificações que nossos órgãos podem adquirir, de acordo, com certos hábitos de vida. É de suma importância que a população como um todo, tenha conhecimento do funcionamento biológico do corpo humano e de como certas patologias podem alterar esse funcionamento. Uma das principais intenções do projeto é que com o conhecimento adquirido os alunos possam praticar hábitos mais saudáveis e consequentemente incentivar a prevenção de diversas doenças prevalentes na sociedade.

\section{Materiais e Métodos}

Foram realizadas visitas mensais em uma escola estadual do município de Vassouras, RJ, Colégio Estadual Centenário com a participação de 20 alunos da Liga nas atividades e desenvolvidas as seguintes etapas: 1) Reunião com a diretoria da Escola; 2) Reunião para definição das etapas do projeto; 3 ) Início das visitas nas escolas; 4) Aplicação do pré-teste com questões objetivas sobre o sistema abordado, com a duração de cerca de 10 min em turmas de 8 ano do ensino fundamental; 5) Elaboração das aulas práticas sobre os temas abordados com peça do Instituto de Anatomia da Universidade de Vassouras; 6) Aplicação do pós-teste com questões semelhantes ao pré teste e com duração de cerca de 10 min; 7) Dinâmica com peças anatômicas, adesivos dos órgãos, manequim e jogo de perguntas e respostas.

O projeto busca através de aulas práticas abordar cada sistema do corpo humano e doenças associadas ao mesmo. O seguinte estudo tem como objetivo relatar a experiência de alunos graduandos de Medicina, integrantes da Liga Acadêmica de Anatomia Humana Professor Froés da Fonseca (LAAHPFF), na 
participação do projeto de pesquisa e extensão "Anatomia nas Escolas" em relação ao aprendizado anatômico, formação acadêmica, impacto nos estudos, dificuldades encontradas e desenvolvimento de habilidades durante a administração do projeto nas escolas de Vassouras. Enfatizando a importância do projeto que o mesmo possa ser ferramenta institucionalizada nos Cursos de Medicina que ainda não o possui, além de estimular a continuidade dessa atividade em nossa Universidade, demostrando uma dinâmica de aprendizado que contribui com a formação de valores profissionais que envolvam trabalho em grupo.

\section{Relato de experiência}

O projeto "Anatomia nas Escolas" realizado durante o primeiro trimestre de 2019 no Colégio Estadual Centenário de Vassouras (RJ). Foi direcionado aos alunos de $8^{\circ}$ ano deste. Foram realizados três encontros, abordando o Trato Gastrointestinal, Sistema Cardiovascular e o Sistema Respiratório, sendo que a metodologia realizada em todos eles foram a mesma descrita anteriormente. Diante disso, podemos relatar a experiência que acadêmicos, membros da Liga de Anatomia Humana e alunos dessa escola obtiveram, evidenciando a importância da participação de todas as etapas do processo de ensino dos sistemas que foram explanados, a troca de experiência e o trabalho em grupo. Com início no Trato Gastrointestinal, abordamos o trajeto do bolo alimentar desde a boca até o ânus, sendo que em cada órgão, falamos das principais patologias e como preveni-las. A boca, onde o principal órgão é a língua, a faringe, a qual faz a transição da cavidade oral para o esôfago. Este, um órgão muscular, que carreia o bolo alimentar ao estômago, onde o alimento é preparado quimicamente para digestão, sendo posteriormente dirigido ao intestino delgado. $\mathrm{O}$ duodeno, primeira porção do intestino delgado, termina a digestão, recebendo a bile e suco pancreático, rico em enzimas necessárias para digestão de proteínas, carboidratos e lipídios. Sequentemente, o alimento é conduzido ao jejuno e íleo, outras duas porções do intestino delgado, para absorção. Então, começa a formação de fezes e absorção de água no intestino grosso, sendo este dividido em sete porções, até chegar ao reto e canal anal.

Além disso, órgãos anexos como fígado, vesícula biliar, pâncreas e baço, foram informados quanto sua localização e função. Neste sistema, discorremos as principais formas de prevenção das doenças relacionadas a esse, como, combater o tabagismo para prevenir câncer de boca, língua e faringe, além de respostas sistêmicas do organismo como câncer de bexiga e estômago; a relevância de levar a vida em equilíbrio, sem estresse excessivo, para não desencadear úlceras gástricas; obter sempre uma alimentação saudável para evitar distensão gástrica, além de prevenir litíase biliar e esteatose hepática, sendo essa última provocada também pelo uso abusivo do álcool.

$\mathrm{Na}$ segunda visita, apresentamos o sistema cardiovascular, mostrando que o coração possui quatro cavidades, dois átrios e dois ventrículos, sendo essas cavidades irrigadas pelas artérias coronárias, como também abordamos sobre a circulação sistêmica e pulmonar. Dentre as prevenções de saúde e as principais patologias, retratamos a Hipertensão Arterial (HAS), doença prevalente no Brasil, que possui fatores genéticos e sociais para seu desenvolvimento, sendo principalmente devido aos hábitos diários como: sedentarismo, obesidade e uso abusivo de sal nas refeições, podendo esta, se não controlada adequadamente, levar a insuficiência cardíaca. Além disso, discorremos sobre o Infarto Agudo do Miocárdio, que se deve a obstrução das artérias coronárias, tendo como fator de risco a HAS e a dislipidemia, e fator desencadeante o estresse.

$\mathrm{Na}$ terceira visita, conversamos sobre sistema respiratório, descrevendo a via de condução do ar, desde a cavidade nasal, passando por faringe, laringe, traqueia, brônquios direito e esquerdo até chegar aos alvéolos. Levantamos mais uma vez a importância de não praticar o tabagismo, sendo essa, a principal causa do Câncer de pulmão, além disso, tal prática leva ao aumento das infecções desse sistema.

Em suma, em todas as visitas foram compartilhados conhecimentos sobre os sistemas propostos, sendo notório o interesse dos alunos nas aulas, o contentamento diante da exposição das peças cadavéricas do Instituto de Anatomia e o entusiasmo na dinâmica ao final das aulas.

\section{Discussão}

O objetivo do trabalho de extensão é colocar em prática projetos, estudos acadêmicos idealizados por alunos e professores, de forma que cheguem a comunidade afim de transforma lá em um ambiente disseminador do conhecimento. Nesse sentido, a união do ensino, pesquisa e extensão é capaz de modificar o modelo pedagógico tradicional, buscando experiências inovadoras no meio em que vivemos ${ }^{5,8}$.

O projeto de pesquisa e extensão "Anatomia nas Escolas", realizado pela Liga Acadêmica de Anatomia Humana Professor Fróes da Fonseca (LAAHPFF), deseja por meio de um vínculo inovador de ensino propagar com mais clareza o funcionamento do corpo humano, transmitindo aos alunos de colégios públicos do município de Vassouras ferramentas necessárias para explorar o próprio corpo e, assim, adquirir noções básicas de cuidados em saúde. Esse trabalho busca 
através do ensino prático solidificar o conhecimento informativo adquiridos pelos alunos.

De acordo com, os Parâmetros Curriculares Nacionais (PCNs) é fundamental valorizar a participação ativa do aluno durante o processo de aprendizagem. Porém, nota se, que muitas escolas não apresentam os recursos necessários para tal valorização. As atividades práticas nas escolas, ainda são escassas, isso se deve à falta de recursos e investimentos públicos na educação como a garantia de laboratórios de ciências, equipamentos de segurança como: luvas, máscaras e jalecos e professores capacitados tornando, assim, viável a valorização da participação ativa do aluno ${ }^{6}$. Desse modo, é dever da Universidade através de atividades de pesquisa e extensão criar ferramentas para propagar o ensino na comunidade 5 .

O projeto "Anatomia nas Escolas" procura desenvolver ferramentas alternativas para suprir a carência de informações teórico e pratica nas escolas no município de Vassouras. Diante disso, o projeto visa com uso de peças do Instituto de Anatomia da Universidade de Vassouras provocar a curiosidade dos alunos e, dessa forma, facilitar a compreensão do material estudado. A cada mês é abordado um sistema do corpo humano, explorando suas funções e estruturas anatômicas importantes. Além disso, um dos principais objetivos do projeto, é por meio da percepção visual dos órgãos adquirir o conhecimento do que é normal e do que é patológico, uma vez que é utilizado peças cadavéricas com patologias recorrentes na população. Desse modo, ao explorarmos um fígado cirrótico, descrevendo suas principais características anatômicas, o aluno é capaz de compreender com maior facilidade os danos que o alcoolismo pode causar no nosso corpo e, assim, entender como podemos evitar a evolução dessa doença. Por meio do trabalho desenvolvidos nas escolas do município de Vassouras, foi possível observar o entusiasmo dos alunos diante das atividades desempenhadas. Muitos elogiaram o projeto e demonstraram se entusiasmados com o trabalho desenvolvido pela (LAAHPFF).

É evidente a relevância que os trabalhos de educação em saúde causam na comunidade, uma vez que através dessas atividades é possível se estimular a prevenção e o autocuidado em saúde, transformando o meio em que atuamos, afim de diminuirmos os casos de negligência em saúde ${ }^{7}$. O empenho dos graduandos de Medicina fortaleceu o vínculo com os alunos da escola estadual e a aproximação dos futuros médicos com a docência e ainda ratifica a máxima ensinar para aprender.

\section{Considerações finais}

As atividades do projeto "Anatomia nas Escola" busca por meio do ensino prático conscientizar com relação aos benefícios do autocuidado com o nosso corpo, ampliando a comunicação, para que assim, seja possível melhorar os índices de qualidade em saúde no município de Vassouras.O interesse e o entusiasmo dos alunos são um grande estímulo para novos projetos, cabe ressaltar também o empenho dos membros da Liga de Anatomia e a percepção da docência como um novo horizonte.

\section{Referências}

1. Alves VS. Um modelo de educação em saúde para o Programa Saúde da Família: pela integralidade da atenção e reorientação do modelo assistencial. Interface (Botucatu) 2005;9(16):39-52.

2. Delfino MRR, Patrício ZM, Martins AS, Silvério MR. O processo de cuidar participante com um grupo de gestantes: repercussões na saúde integral e individual-coletiva. Ciênc. saúde coletiva 2004;9(4):1057-66.

3. Duarte V, Santos M, Souza E, Borges G. "Brincando com a fisiologia humana": Relato de uma extensão universitária. Rev. Ciênc. Ext 2012; 8, (1):98-106.

4. XIII JORNADA DE ENSINO, PESQUISA E EXTENSÃO. Anatomia humana para todos: contribuindo para a compreensão do corpo humano. Disponível em: <http://www.eventosufrpe.com.br/2013/cd/resumos/r0720-1. pdf $>$. Acesso em: 30 jul. 2017.

5. Ferreira D, Aranha R, Souza MH. Ligas acadêmicas: uma proposta discente para ensino, pesquisa e extensão. Rev Int 2011;(16):47-51.

6. BRASIL, Ministério da Educação, (1997). Parâmetros Curriculares Nacionais para o Ensino Fundamental. Brasília, MEC/SEF.

7. Fernandes K, Claro M, Firmeza S, Andrade C, Souza A, Silva A Relato de experiência: vivências de extensão na comunidade. Rev Ciênc Ext 2016;12(1):97-104.

8. Oliveira F, Ferreira A, Mota B, Machado M. A busca pela qualidade educacional: avaliação das práticas lúdicas relacionadas ao ensino de anatomia humana através da interdisciplinaridade na formação de docentes. In: Anais do 23. Seminário Internacional de Educação, Tecnologia e Sociedade; 2018 nov 06-16:FACCAT. p. 1-10. 